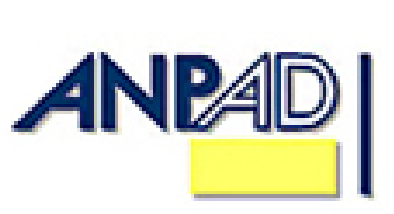

Disponível em

http://www.anpad.org.br/rac

RAC, Curitiba, v. 14, n. 4, art. 8,

pp. 703-721, Jul./Ago. 2010

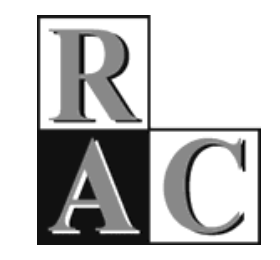

\title{
O Impacto da Conexidade no Comportamento do Consumidor em Relação aos Programas de Televisão
}

The Impact of Consumer Behavior Connectedness on Television Shows

Valter Afonso Vieira* Doutor em Administração de Empresas pela UnB. Professor no CEPPAD/UFPR, Curitiba/PR, Brasil.

Jucelia Appio Doutoranda em Administração pela Universidade Positivo (UP). Professora da Faculdade Horus, Pinhalzinho/SC, Brasil.

* Endereço: Valter Afonso Vieira

Rua Viuvinha, 69, Arapongas/PR, 86709-340. E-mail: valterafonsovieira@yahoo.com

Copyright (C) 2010 RAC. Todos os direitos, inclusive de tradução, são reservados. É permitido citar parte de artigos sem autorização prévia desde que seja identificada a fonte. 


\section{RESUMO}

O presente artigo buscou verificar as propriedades psicométricas da escala de conexidade com programas de TV no comportamento do consumidor. Consumidores responderam às questões do instrumento com relação a programas de televisão. Utilizando análises exploratória, confirmatória, discriminante e nomológica, foi possível confirmar cinco das seis dimensões inicialmente propostas pela escala. Os resultados do trabalho também confirmaram a relação significativa e positiva entre conexidade e envolvimento com programas de TV, entre conexidade e assistir TV no geral, e entre conexidade e comunidade de marca. Conexidade e interação social com envolvimento tiveram uma relação negativa.

Palavras-chave: escala; TV; conexidade; comportamento do consumidor.

\section{ABSTRACT}

This paper analyzes the psychometric proprieties of the connectedness scale on consumer behavior. Consumers answered questions relating to television shows. Using exploratory, confirmatory, discriminant, convergent and nomological analyses, we found support for five dimensions out of the six initially proposed to reflect the scale. Furthermore, the results supported a positive relationship between connectedness and involvement, connectedness and hours watching TV and connectedness and brand community. Connectedness and social interaction were found to have a negative relationship.

Key words: scale; connectedness; TV; consumer behavior. 


\section{INTRODUÇÃO}

De acordo com Kagami (2006), os programas a cores de televisão foram iniciados no Brasil em 1972. Com o passar dos anos, a televisão consolidou-se como ferramenta de educação, informação e entretenimento, servindo para diversos propósitos. No que tange ao entretenimento, programas de diversão - muitas vezes chamados de produtos pelas emissoras - se espalharam pelos canais abertos. Esses programas variam desde novelas, esportes, mini-séries, comédias, reality shows, programas investigativos até jogos de participação interativa por telefone, cartas e Internet. Devido à consequente extensão de produtos, nota-se que a televisão brasileira tem um potencial de influenciar a vida das pessoas.

De fato, o poder da televisão na camada social faz com que haja necessidade de pesquisas, objetivando verificar a extensão na qual as pessoas desenvolvem relações específicas com programas de TV e como essas associações afetam as suas experiências de vida. Nesse contexto, pode-se dizer que os consumidores possuem ligações com os programas, associações que variam desde um pequeno interesse até uma enorme conexidade, quando se chega ao ponto de telespectadores copiarem características dos atores para a vida real (ex. Copiar estilos e modos de vestir).

Portanto investigar as relações dos indivíduos com os programas de TV torna-se uma necessidade. Além do mais, verificar teoricamente se existe um construto para avaliar o fenômeno de consumo excessivo dos programas de TV é de suma importância para a academia de marketing. Sob essa circunstância, a literatura de comportamento do consumidor propõe a conexidade - qualidade do conexo - com programas de TV como uma variável latente (Russell \& Puto, 1999), a qual explica padrões de comportamento. Não obstante, esse construto requer testes de validade, confiabilidade e convergência, para ser mais bem consolidado no campo do marketing (Vieira \& Slongo, 2007). Diante dessa necessidade, este artigo busca verificar a escala de conexidade com programas de TV ${ }^{(1)}$ de Russell, Norman e Heckler (2004).

Justifica-se estudar a extensão na qual as pessoas desenvolvem relações com os programas de TV e como essas relações afetam as suas experiências de consumo, pois, de certo modo se estaria descobrindo necessidades pelas quais as pessoas gastam horas e horas na frente dos programas. Isso pode auxiliar as emissoras de TV a criarem estereótipos nos shows que satisfaçam às necessidades dos consumidores. Por outro lado, as empresas podem utilizar propagandas dentro dos programas para alavancar vendas. Além do mais, justifica-se este trabalho pelo fato de que ele auxilia na avaliação de um instrumento útil para psicólogos verificarem um consumo.

Para verificar a escala, o artigo está estruturado da seguinte forma: a primeira parte faz uma revisão de literatura sobre o construto conexidade, de modo a ressaltar sua origem e definição teórica. Após essa etapa, quatro proposições de pesquisa são apresentadas, visando comprovar a validade nomológica. Na próxima parte é apresentado o método de pesquisa. Na etapa seguinte os dados são analisados e discutidos. Considerações finais e contribuições científicas concluem o artigo.

\section{DEFINIÇÃo CONCEITUAL}

A programação da televisão gera experiências simples para entretenimento diário, mas por vezes estranhas para os telespectadores. No que tange a essas últimas, por exemplo, Maccoby e Wilson (1957) sugerem que telespectadores chegam a internalizar as experiências vividas pelas personagens da TV na vida real. Isso significaria uma associação tão grande com a personagem que o telespectador começa a incorporar partes do show em sua vida real. Levy (1962) sugere que, ao assistir televisão, existe o desenvolvimento de uma relação parassocial dos telespectadores com as personagens. Nesse sentido, a interação experimentada pelo telespectador com seu programa favorito pode gerar uma maior rede de amigos (Russell et al., 2004), que também assistem ao mesmo programa. 
Reconhecendo as limitações das medidas de audiência de TV e visando preencher essa lacuna, Russell e Puto (1999) sugeriram o construto conexidade como indicador da natureza e da intensidade da relação entre o telespectador e um programa de TV. A proposta desses autores é que o construto represente uma transcendência ao simples fato de assistir à programação da televisão, caracterizando a construção da identidade social e do próprio self de quem assiste aos programas (Belk, 1988, 1992). Nessa linha de raciocínio, ter conexidade com programas de TV significa ter uma fissura extrema pela programação oferecida pela telinha, onde o indivíduo escolhe um programa predileto e o consome frequentemente, incorporando e se deixando incorporar por fenômenos que envolvem a programação, como roupas, estilos, objetos, dentre outros elementos.

Por consequência do surgimento desse fenômeno em comportamento do consumidor, Russell et al. (2004), avançando nos achados de Russell e Puto (1999), identificaram a necessidade de desenvolver um instrumento para avaliar a conexidade e inseri-la em uma rede nomológica. Definindo a variável, Russell et al. (2004) conceitualizam conexidade como "o nível de intensidade da relação que o telespectador desenvolve com as personagens e padrões contextuais de um programa no ambiente televisivo parassocial" (p. 152).

Diante de tais condições surgiu a escala de conexidade com programas de TV, a qual possui seis fatores $^{(2)}$ Essas dimensões representam as diferentes manifestações de como os telespectadores se associam com os programas de TV e de como eles desenvolvem relações parassociais com as personagens. Seus 85 itens foram gerados por meio de três grupos de foco com duração de uma hora cada: grupos com variação de $N=5$ até $N=9$ pessoas. Após uma análise prévia dos indicadores, restaram 45 itens, os quais foram verificados quantitativamente em uma amostra de $N=175$ estudantes. Portanto da análise fatorial final surgiu a escala de conexidade com programas de TV, composta de seis dimensões e dezesseis indicadores respectivos.

Russell et al. (2004) assim explicam os fatores propostos: o fator Fuga se caracteriza pelo elemento catártico, que conecta o telespectador com o programa. Na verdade, essa dimensão sugere que as pessoas assistem aos programas de TV para escapar da realidade do mundo, buscando esquecer, por momento, seus problemas particulares. $\mathrm{O}$ fator Modelar avalia o processo de aprendizagem social no qual os indivíduos relatam suas próprias experiências com aquelas demonstradas pela programação. Por exemplo, telespectadores se relacionam com determinadas personagens e, após isso, modelam suas vidas de modo similar àquelas. Salienta-se que esse fator, na verdade, está relacionando os aspectos afetivos dos indivíduos com os programas da televisão. O fator Moda analisa como um telespectador é influenciado pela aparência do protagonista de determinado programa. Busca-se, portanto, copiar roupas, estilos de vida e cortes de cabelo de personagens. Imitação avalia a inclinação das pessoas em imitar as personagens, provavelmente devido aos estímulos emocionais derivados de outro papel. Na prática, essa dimensão traduz aspectos como imitar palavras, tiques, vozes e barulhos dos caracteres. O fator Aspiração identifica como as pessoas se tornam conexas com um programa de TV no qual elas aspiram a um dia estar no show ou encontrar sua personagem principal. Por exemplo, "eu poderia ser um dia um ator (atriz) no...". Por fim, o fator Parafernália verifica o grau no qual as pessoas obtêm itens/objetos relacionados aos programas de TV e os utilizam no mundo real, tais como revistas, livros e pôster.

Apresentados o construto e a dimensionalidade da escala, as proposições de pesquisa são apresentadas na sequência. A construção das proposições visa relacionar ou não o construto com outros, que podem ser (ou não) similares. Essas proposições auxiliam na validade externa do construto que está sendo estudado por meio da rede nomológica (Peter, 1981). Churchill (1996) sugere que um instrumento tem validade nomológica "quando ele se comporta como o esperado com relação a outro construto que é teoricamente relacionado ou não" (p. 538). 


\section{PROPOSIÇÕES DE PESQUISA}

Atitude junto ao Programa de TV. O construto atitude tem sido utilizado extensivamente para refletir algum grau, a favor ou não, de atitude junto a um objeto (Cacioppo, Gardner, \& Berntson, 1997). Que atitude o cliente tem diante de um programa de televisão? Você é a favor ou não de um programa $x$ ? A atitude busca fornecer respostas a questões como esta. Para Engel, Blackwell e Miniard (2000), atitude é "uma avaliação geral que pode variar de extremamente positiva a extremamente negativa" (p. 617), ou seja, não é um comportamento em si. Medidas gerais de atitude têm sido utilizadas para examinar o efeito dos programas de TV sobre as avaliações dos telespectadores e podem ser caracterizadas como modos de avaliação de programas (Barwise \& Ehrenberg, 1987) ou estimuladores de emoção (Murry, Lastovicka, \& Singh 1992). Assim, tais trabalhos verificam até que ponto (a favor ou contra) o telespectador avalia o programa de TV. Russell et al. (2004) propõem que o comportamento associado à conexidade vai além das respostas gerais avaliativas de programas de TV e, portanto, é distinto da atitude. $\mathrm{O}$ argumento sugere que uma atitude extremamente positiva com a programação de TV é apenas um passo inicial para chegar à conexidade. A atitude em si não seria capaz de capturar uma interação parassocial (Levy, 1962), enquanto uma pessoa com alta conexidade com programas de TV pode ter uma relação mais profunda e mais íntima com as personagens, podendo interagir mais nas suas relações sociais (Russell et al., 2004). Sob essas circunstâncias, os consumidores possuem atitudes positivas com diversos programas de TV (ex. Esportes, domingo pela tarde, novelas etc.); mas esse favorecimento não chega ao ponto de induzir um comportamento de interação parassocial, gerada pelo construto conexidade. Este último teria o poder de gerar pontos de encontro ou blogs, em que as pessoas poderiam discutir detalhadamente aspectos dos programas. Desse modo, a primeira proposição elaborada é:

$\mathbf{P}_{1 \mathbf{a}}$ : Conexidade com programas de TV e Atitude junto aos programas de TV são associados positivamente, porém $\left(\mathrm{P}_{1 \mathrm{~b}}\right)$ são construtos distintos .

Envolvimento. Para fins deste artigo, envolvimento é "a importância percebida de uma pessoa para com um objeto baseado em interesse, valores e necessidades inerentes" (Zaichkowsky, 1985, p. 342). Dholakia (1997) vê o envolvimento como condição motivadora, definida como "importância pessoal percebida de um produto para um indivíduo, baseada em suas necessidades, valores e interesses" ( $p$. 169). Existem sugestões de que as pessoas assistem aos programas de TV por possuírem certo grau de envolvimento (Park \& McLung, 1986). Russell et al. (2004) propõem que conexidade com programas de TV e envolvimento são construtos distintos e separados, baseando-se no fato da distinção na relação entre a marca-consumidor e a compra repetida. Em outras palavras, o consumidor pode possuir uma relação com a marca de muita afinidade, mas mesmo tendo tal associação a compra repetida não ocorreria necessariamente. Dado o exemplo, o argumento é que programas de TV começam fomentando o envolvimento do telespectador; todavia, com o passar de vários episódios, os programas acabam absorvendo demasiadamente a atenção daquele show para uma relação afetiva com os caracteres no programa de TV (Fournier, 1998). Essa nova relação seria uma afinidade que foge do envoltório do envolvimento e entra nas delimitações de outro fenômeno - no caso a conexidade - uma vez que a interação tende a ser maior. Se esse argumento está correto, espera-se que envolvimento e conexidade sejam diferentes, porém associados positivamente. Além do mais, envolvimento é atrelado à propaganda (Macinnis \& Whan, 1991), à mensagem (Krugman, 1977), ao produto (Laurent \& Kapferer, 1985), à marca, ao meio ou à decisão (William, 1993), mas conexidade não, sendo portanto diferentes. Terceiro, Richins e Bloch (1986) sugerem que envolvimento é dividido em situacional somente para situações específicas, tais como a compra - e duradouro - com relação a um tempo maior de relação com o produto. Conexidade não possui esta divisão e portanto deve ser diferente de envolvimento. Desse modo, a próxima proposição de pesquisa é:

$\mathbf{P}_{2 \mathrm{a}}$ : Conexidade com programas de TV e Envolvimento junto ao programa de TV são associados positivamente, porém são construtos $\left(\mathrm{P}_{2 \mathrm{~b}}\right)$ distintos. 
Conexidade com programas de TV e Assistir TV no geral. O montante assistido de TV (ex. Tempo/horas) é comumentemente utilizado como medida da influência da TV. Uma criança americana média terá assistido a 200.000 atos violentos e 16.000 assassinatos na TV até os 18 anos; adolescentes que assistiam, quando crianças, a programas com temas de adultos na TV, tornaram-se sexualmente ativos mais cedo que adolescentes da mesma idade que não assistiam a estes programas (Oliveira, Sanvitto, Gus, \& Zelmanowicz, 2009). Logo, se a influência do programa de TV realmente é eficaz, o montante geral de televisão assistido será maior. Todavia essa medida não auxilia a caracterizar a relação seletiva que consumidores têm com programas específicos. Esta medida representaria mais uma idéia de frequência global, onde audiência e atenção são colocadas como elementos diretamente proporcionais. Entretanto a idéia é outra; conexidade com programas de TV e assistir TV no geral são construtos ortogonais (Russell et al., 2004). Telespectadores assíduos podem conectar-se a vários programas ao mesmo tempo ou a apenas alguns. Isso quer dizer, muito tempo é desprendido assistindo TV, mas não tanto em profundidade. Por exemplo, telespectadores light podem assistir raramente televisão, mas quando o fazem eles são altamente fissurados e aprofundados em um programa específico. De outro modo, outros consumidores assistem horas e horas a TV, mas não sabem ao certo o que foi assistido. Portanto, mesmo assistindo muitas horas, o consumidor pode não estar conexo com o programa. Consequentemente, deveria existir discriminação entre assistir muita TV e conexidade. Logo:

$\mathbf{P}_{3 \mathrm{a}}$ : Conexidade com programas de TV e Assistir TV no geral são associados positivamente, porém são construtos $\left(\mathrm{P}_{3 \mathrm{~b}}\right)$ distintos.

Interação Social. Acredita-se que o grau de conexidade com programas de TV gere algumas consequências sociais nos telespectadores. Pesquisas têm mostrado que programas de TV podem contribuir para uma identidade social do indivíduo e aumentar a comunidade de colegas (Kozinets, 2001; Russell \& Puto, 1999). Nessa conjuntura, presume-se que quanto mais um telespectador se torna vinculado com o programa, $\left(\mathrm{P}_{4 a}\right)$ mais ele aumentará a interação social com colegas, incrementando a rede de relacionamentos com outras pessoas que assistem ao mesmo show. O consumidor deveria sentir necessidade de conversar e discutir com amigos sobre o mesmo programa. Logo, $\left(\mathrm{P}_{4 \mathrm{c}}\right)$ essa conversa com uma pessoa deveria sugerir novas (outras) pessoas que também assistem ao programa, aumentando o tamanho da rede de relacionamento (colegas). Por fim, $\left(\mathrm{P}_{4 \mathrm{~b}}\right)$ quanto mais conexidade com programas de TV, mais o indivíduo tende a consumir a comunidade de marca do programa - ou seja, gostar, idolatrar e adorar a programação e/ou objetos relacionados ao show (McAlexander, Schouten, \& Koening, 2002). Desse modo:

$\mathbf{P}_{4}$ : Quando a conexidade com programas de TV aumenta, aumentam também a $\left(\mathrm{P}_{4 \mathrm{a}}\right)$ interação social com outras pessoas, a $\left(\mathrm{P}_{4 b}\right)$ relação com a comunidade de marca e o $\left(\mathrm{P}_{4 \mathrm{c}}\right)$ tamanho da rede de colegas que assistem ao mesmo programa.

Um resumo da proposta de exame psicométrico da escala de conexidade com programas de TV está apresentado na Tabela 1.

Tabela 1

\section{Estrutura Teórica do Trabalho}

\begin{tabular}{|l|l|}
\hline \multicolumn{1}{|c|}{ Exame Psicométrico } & \multicolumn{1}{|c|}{ Hipótese Proposta } \\
\hline Fatores de Conexidade & Conexidade possui seis fatores \\
\hline Validade Convergente & $\begin{array}{l}\mathrm{P}_{1 \mathrm{a}} \text { : Conexidade e atitude são associadas positivamente } \\
\mathrm{P}_{2 \mathrm{a}} \text { : Conexidade e envolvimento são associados positivamente } \\
\mathrm{P}_{3 \mathrm{a}} \text { : Conexidade e assistir TV no geral são associados positivamente }\end{array}$ \\
\hline Validade Discriminante & $\begin{array}{l}\mathrm{P}_{1 \mathrm{~b}} \text { : Conexidade e atitude são distintas } \\
\mathrm{P}_{2 \mathrm{~b}} \text { : Conexidade e envolvimento são distintos } \\
\mathrm{P}_{3 \mathrm{~b}} \text { : Esperança e assistir TV no geral são distintos }\end{array}$ \\
\hline Poder Preditivo & $\begin{array}{l}\mathrm{P}_{4 \mathrm{a}, 4 \mathrm{~b}, 4 \mathrm{c}} \text { : Conexidade e interação social, comunidade de marca e tamanho da rede } \\
\text { são associados positivamente }\end{array}$ \\
\hline
\end{tabular}




\section{MÉTODO}

Como o objetivo do artigo é analisar a escala de conexidade, escolheu-se um levantamento de pesquisa do tipo descritivo (Churchill, 1996). Inicialmente, a escala foi traduzida para o português por dois acadêmicos de marketing proficientes no idioma. Posteriormente cada item foi traduzido de volta para um aluno de doutorado da Universidade da Flórida. Por consequência, a escala foi pré-testada com nove pessoas em três pré-testes diferentes, ou seja, três pessoas em cada pré-teste. Os pré-testes foram em estilo de entrevista de profundidade, visando encontrar problemas de compreensão e significados (Malhotra, 2000). Na seqüência, passou-se para a etapa quantitativa, na qual uma amostra por conveniência foi utilizada (Churchill, 1996).

A amostra foi caracterizada como não-probabilística por cotas (Aaker, Day, \& Kumar, 2005), escolhida por meio do sorteio de bairros de uma cidade. Um bairro foi sorteado aleatoriamente e, após esse processo, escolheu-se aleatoriamente a primeira casa do respectivo bairro e se iniciou a pesquisa. Os dados foram coletados de maneira pessoal (Churchill, 1996); o pesquisador fazia as indagações e o respondente dava sua resposta. Desse modo, foram investigadas residências em sequência, até um total de 127 questionários. A literatura sugere que para o tamanho da amostra, cinco observações são necessárias para cada item do questionário (Hair, Anderson, Tatham, \& Black, 1998). Tomando por base esse critério, um mínimo de 80 observações seria necessário. Portanto o número final de observações, saturando as 80 , foi o suficiente para o emprego das técnicas multivariadas, pois a relação foi de oito observações por item.

No que tange às medidas na pesquisa, o questionário foi composto por 16 itens da escala conexidade com programas de TV de Russell et al. (2004), variando de Concordo Totalmente/Discordo Totalmente. Sete itens da escala da Comunidade de Marca foram retirados do artigo de McAlexander et al. (2002). Esse instrumento objetivou verificar o quanto as pessoas gostavam, amavam, indicavam e adoravam seu programa predileto $\left(\mathrm{P}_{4 \mathrm{~b}}\right)$; variando de Concordo Totalmente/Discordo Totalmente. Cinco itens escolhidos aleatoriamente da escala de Zaichkowsky $(1985)\left(\mathrm{P}_{2}\right)^{(3)}$ foram empregados para avaliar o Envolvimento. Dois itens foram utilizados para aferir a Interação Social do telespectador $\left(\mathrm{P}_{4 \mathrm{a}}\right)$, sendo 'quão frequente você fala do programa de TV para outros?' e 'quão frequente você assiste ao seu programa de TV favorito com outros?', variando de Frequentemente/Não Frequentemente. Três itens foram utilizados para verificar Atitude com o programa de TV, variando de Eu Gosto/Não Gosto; Bom/Mal; Agradável/Desagradável $\left(\mathrm{P}_{1}\right)$. Uma questão de assistir TV no geral avaliava 'quantas horas por semana o telespectador gastava assistindo ao programa favorito? _ ' $\left(\mathrm{P}_{3}\right)$. Por fim, uma questão indagava 'quantas pessoas você conhece que assistem ao mesmo tipo de programa de TV? ,$\left(\mathrm{P}_{4 \mathrm{c}}\right)$. Todas as perguntas continham variações de cinco pontos. Detalhes estão no Anexo I e II.

Uma limitação do artigo está no uso de uma amostra não-probabilística, fazendo com que os dados não sejam plausíveis de generalização para a população como um todo. Outra limitação é que os dados não são do tipo longitudinal; assim, comparações longitudinais dos resultados não são possíveis de serem realizadas.

\section{ANÁLISE DOS DADOS}

No total 127 pessoas responderam à pesquisa. Quanto ao sexo, 60\% (100) dos respondentes eram do sexo feminino. No geral, os respondentes conheciam uma média de 30 pessoas que assistiam ao mesmo programa de TV que eles (variação 1 até 400). No que tange ao número médio de episódios novos por mês, os telespectadores assistiam a 18 episódios (variação 2 até 30). Por fim, eles gastavam em média 8 horas por semana assistindo àquele tipo de programação específico. 
Com relação ao tipo/nome de programa, a Tabela 2 apresenta os resultados. Em questões gerais relativas à classificação de assistir TV, a novela obteve aproximadamente $55 \%$ dos votos (acumulado), jornais e programas de realidade nacional obtiveram $21 \%$ e outros $24 \%$. Nota-se um disparo muito grande da novela por parte de $71(56 \%)$ observações.

Tabela 2

Classificação dos Programas

\begin{tabular}{lcccc}
\hline Programa & Frequência & \% & \%Válida & \%Acumulada \\
\hline Jornal Nacional & 21 & 16,5 & 16,5 & 16,5 \\
Malhação & 4 & 3,1 & 3,1 & 19,7 \\
Rebeldes & 10 & 7,9 & 7,9 & 27,6 \\
Belíssima & 24 & 18,9 & 18,9 & 46,5 \\
Alma Gêmea & 14 & 11,0 & 11,0 & 57,5 \\
Bang Bang & 9 & 7,1 & 7,1 & 64,6 \\
Escrava Isaura & 4 & 3,1 & 3,1 & 67,7 \\
Novelas (outras) & 6 & 4,7 & 4,7 & 72,4 \\
Show da Fé & 8 & 6,3 & 6,3 & 78,7 \\
Globo Esporte & 7 & 5,5 & 5,5 & 84,3 \\
Outros/Gerais & 20 & 12,7 & 12,7 & 100,0 \\
Total & 127 & 100,0 & 100,0 & \\
\hline
\end{tabular}

Após as análises iniciais, os dados foram verificados em termos de valores em branco. Devido ao processo de coleta de dados pessoais, nenhum valor em branco foi obtido, demonstrando a vantagem da coleta pessoal. A seguir, os dados foram submetidos à análise dos valores extremos, seguindo o procedimento sugerido por Hair et al., (1998) “de (a) valor escore $Z$ acima de módulo 3 e (b) cálculo da distância de Mahalanobis e exclusão dos casos com valores de significativos" (p. 69).

Seguindo esse procedimento, nenhum caso foi identificado. Uma análise na normalidade univariada, pressupondo que os dados são normais, foi executada (ex. testes Kolmogorov Smirnov e comparação de gráficos Dretended Normal Q-Q e Gráfico Q-Q). Assim, foram empregados procedimentos de análise, considerando os valores de assimetria e de curtose. Os valores achados estiveram dentro de parâmetros considerados moderados - aqui assumidos como assimetria $\leq 2$ e curtose $\leq 7$, conforme sugestão de West, Finch e Curran (1995). Os dados foram considerados não normais.

Dando prosseguimento, verificou-se a multicolinearidade, buscando averiguar se as variáveis eram diferentes ou se mediam a mesma coisa (Malhotra, 2000). Em outras palavras, isso significa que não podem existir correlações altas entre si (maior de $r \pm 0,90$ ), valor esse indicado pela literatura (Hair et al., 1998). Os resultados da matriz de correlação de Pearson não advertiram a multicolinearidade. Todavia existem outros métodos para verificar multicolinearidade, que podem ser melhores do que a correlação de Pearson, como por exemplo, o Fator de Inflação da Variância [VIF] e a Tolerância [T]. A literatura assinala que existe indicação de multicolinearidade, se o valor do VIF, encontrado na análise de regressão, for maior do que 10 ou T maior do que 6 (Hair et al., 1998). Para tal fim, uma análise de regressão entre a combinação dos 16 itens foi feita (dentre os itens). Os resultados do VIF e $\mathrm{T}$ estiveram todos dentro dos parâmetros esperados e, portanto, não indicaram a presença de colinearidade. Nesse sentido, segundo as boas evidências dos dados, passou-se para uma análise da estrutura do instrumento.

Análise Fatorial Exploratória. A verificação da unidimensionalidade da estrutura foi feita por meio da análise fatorial exploratória com o método dos componentes principais e a rotação varimax. As cargas fatoriais foram retidas apenas em caso de $\lambda>0,40$ (Costello \& Osborne, 2005). Esperava-se uma estrutura de seis fatores, indicando os seis construtos propostos. Após a realização da análise fatorial 
três variáveis tiveram cargas fatoriais em dimensões não correspondentes teoricamente com suas propriedades. Elas foram: Moda 3, Aspiração 1 e Aspiração 2 (ver Anexo I). Obviamente, retiraramse tais variáveis (uma por vez) e novas rotações foram feitas. A Figura 1 mostra a discrepância e distorção de opinião nos dois indicadores de aspiração, invalidando assim a dimensão.
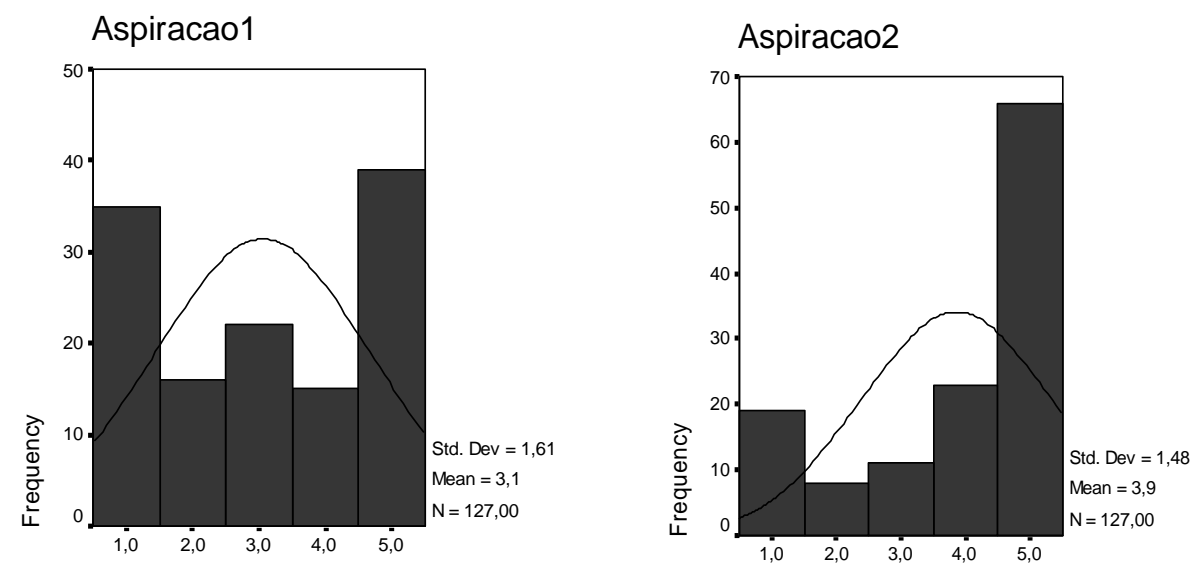

Figura 1. Histograma das Variáveis do Construto Aspiração

Optou-se pela exclusão da dimensão aspiração do instrumento conexidade com programas de TV. Isso explica que a amostra entrevistada não percebeu uma necessidade de aspiração de estar no show ou de encontrar a personagem preferida. No momento, essa dimensão indicaria supostamente uma cobiça do telespectador de estar em determinados shows fazendo o papel de protagonista junto a celebridades, tais como Silvio Santos, Luciana Gimenez, Eliane, Luciano Huck, Hebe Camargo etc.

Em outras palavras seria uma maneira de desejar estar/ser com o ídolo. Uma possível explicação para eliminação do fator aspiração é o fato de no Brasil existir uma carência de programas em que os telespectadores participam ativamente do desenvolvimento do show. Hebe Camargo, Jô Soares e De Frente com Gabi são programas mais para pessoas públicas conhecidas (ex. celebridades). Portanto, faltaria uma identidade maior do telespectador para com o desejo de estar no palco, como é feito no programa do Netinho e do Raul Gil.

De acordo com a Tabela 2, a estrutura final da escala apresenta uma variância explicada de 66,6\%, com autovalores iguais a 3,35 (25\% variância explicada); $1,56(12 \%) ; 1,46(11 \%) ; 1,18$ (9\%) e 1,09 (8\%), respectivamente. Contudo é importante observar que o método de extração pelo autovalor, muitas vezes, erra na estimação do número correto de fatores em $80 \%$ das vezes versus $20 \%$, quando se utiliza a análise paralela (Zwick \& Velicer, 1986). Nesse contexto, o programa RanEigen Syntax foi utilizado para determinar o número de fatores (Enzmann, 1997; Vieira, 2009). Diferentemente do encontrado pela Tabela 2, o resultado da análise paralela indicou apenas três componentes, ao invés de cinco. Esse resultado será confirmado adiante por meio da confiabilidade. Outras informações descobertas foram: Kaiser Meyer Olkin e o teste de Bartlett indicou valores de 0,71 para o primeiro e $\chi^{2}=356,158 ;$ g.l. $=78 ; p<0,001$ para o segundo, estando dentro dos níveis indicados (Malhotra, 2000).

Tabela 3

Matriz Rotacionada da Escala de Conexidade

\begin{tabular}{|c|c|c|c|c|c|}
\hline & \multicolumn{5}{|c|}{ Fatores } \\
\hline & 1 & 2 & 3 & 4 & 5 \\
\hline Escape 2 & ,824 & $5,098 \mathrm{E}-02$ & $1,024 \mathrm{E}-2$ & $-1,23 \mathrm{E}-02$ & ,318 \\
\hline Escape 3 & ,790 &, 790 & $6,943 \mathrm{E}-02$ &, 110 & $-3,48 \mathrm{E}-02$ \\
\hline
\end{tabular}

Continua 
Tabela 3 (continuação)

\begin{tabular}{lrrrrr}
\hline & \multicolumn{7}{c}{ Fatores } \\
\cline { 2 - 7 } & $\mathbf{1}$ & $\mathbf{2}$ & $\mathbf{3}$ & \multicolumn{1}{c}{$\mathbf{4}$} & \multicolumn{1}{c}{$\mathbf{5}$} \\
\hline Escape1 & $\mathbf{7 5 4}$ &, 167 &, 114 & $-5,61 \mathrm{E}-04$ & $-8,65 \mathrm{E}-03$ \\
Modelo 2 &, 122 & $\mathbf{, 8 1 0}$ &, 161 & $9,113 \mathrm{E}-02$ & $5,767 \mathrm{E}-02$ \\
Modelo 3 & $5,250 \mathrm{E}-02$ & $\mathbf{8 1 0}$ & $-8,01 \mathrm{E}-03$ &, 226 & $-3,06 \mathrm{E}-03$ \\
Modelo 1 &, 246 & $\mathbf{, 6 1 3}$ &, 142 & $-1,88 \mathrm{E}-02$ &, 211 \\
Fashion 2 & $-6,19 \mathrm{E}-03$ & $3,830-03$ & $\mathbf{, 8 7 8}$ & $-1,64-02$ &, 131 \\
Fashion 1 &, 154 &, 214 & $\mathbf{, 8 1 2}$ & $8,289 \mathrm{E}-02$ & $-7,37 \mathrm{E}-02$ \\
Imitação 1 & $4,752 \mathrm{E}-02$ & $5,621 \mathrm{E}-02$ & $3,370 \mathrm{E}-02$ & $\mathbf{8 3 4}$ & $-9,69 \mathrm{E}-03$ \\
Imitação 3 & $-3,28 \mathrm{E}-02$ &, 138 & $-3,18 \mathrm{E}-03$ & $\mathbf{, 7 0 8}$ &, 221 \\
Imitação 2 &, 160 &, 159 &, 439 & $\mathbf{4 8 0}$ & $1,827 \mathrm{E}-02$ \\
Parafernália 2 & $-9,51 \mathrm{E}-03$ &, 216 &, 145 & $-1,42-02$ & $\mathbf{8 0 2}$ \\
Parafernália 1 &, 201 & $-2,24 \mathrm{E}-02$ & $-8,58 \mathrm{E}-02$ &, 266 & $\mathbf{, 7 6 4}$ \\
\hline
\end{tabular}

Confiabilidade. Para avaliar a confiabilidade da escala utilizada foi adotado o método de consistência interna. Neste contexto, esperava-se que todas as dimensões da escala apresentassem valores alphas $>0,70$ (Malhotra 2000). Os valores de confiabilidade encontrados foram $\alpha=0,74$ para Fuga; $\alpha=0,69$ para Modelo; $\alpha=0,71$ para Moda; $\alpha=0,54$ para Imitação e $\alpha=0,53$ para Parafernália. Inicialmente, nota-se a baixa confiabilidade das dimensões Imitação e Parafernália. Portanto corrobora-se aqui o resultado de sugestão de três fatores com aquele encontrado com a análise paralela. Verificações adicionais de confiabilidade composta $(\mathrm{CR})$ e da média da variância extraída (AVE) dos construtos foram feitos, onde a literatura indica valores ideais sendo maiores do que $0,70 \mathrm{e}$ 0,50 para os mesmos (Fornell \& Larcker, 1981).

Para confiabilidade composta os dados sugeriram os seguintes números: Fuga $(0,75)$ Modelo $(0,70)$; Moda $(0,76)$; Imitação $(0,54)$ e Parafernália $(0,53)$ e para média de variância extraída os valores foram: Escapar (0,50); Modelo $(0,44)$; Moda $(0,52)$; Imitação $(0,44)$ e Parafernália $(0,36)$. De modo geral, de acordo com esse segundo critério de confiabilidade, os construtos Imitação e Parafernália demonstraram baixa confiabilidade dos dados e, portanto, deveriam ser revistos em pesquisas futuras. Em resumo, dada a existência e comprovação de uma estrutura multidimensional da escala com cinco fatores, a perda de uma dimensão (Aspiração) e a baixa confiabilidade de dois conceitos (Parafernália e Imitação), visou-se à verificação da validade convergente.

Validade Convergente. A análise da validade convergente indica se as medidas de um construto estão suficientemente relacionadas aos construtos latentes de interesse (Malhotra, 2000). Bagozzi e Philips (1982) propõem a análise fatorial confirmatória como procedimento para fazer a avaliação da validade convergente dos construtos. Os critérios sugerem que seja verificada a significância das cargas fatoriais dos construtos em nível de $5 \%$ ou $1 \%$, onde o $t$ crítico corresponde a $1,65(\alpha=0,05)$ ou a $2,236(\alpha=$ $0,01)$. Os resultados indicaram a maioria das cargas significativas a $p<0,001$. A validade convergente da estrutura de conexidade com programas de TV foi comprovada em todos os itens.

Análise Fatorial Confirmatória. No modelo de análise fatorial confirmatória (Arbuckle, 1997), quatro opções de estrutura concorrentes foram criadas e comparadas. A primeira (A) é um modelo de segunda ordem, cujas cinco dimensões foram associadas ao construto final conexidade. A outra opção (B) foi elaborada na forma de cinco fatores de primeira ordem, permitindo a correlação entre eles. A próxima opção (C) foi desenhada de forma que todos os itens foram ligados a um único fator geral. A última versão foi a de três fatores indicados pela análise paralela, permitindo correlações entre os construtos (D). Todas as estruturas foram examinadas, empregando a estimativa de máxima verossimilhança. A escolha do melhor modelo recaiu sobre o qui-quadrado $\left(\chi^{2}\right)$ por graus de liberdade (g.l.), pois é a medida mais fundamental de ajuste geral (Arbuckle, 1997). Baixos valores de qui-quadrado indicam que as matrizes de entrada real e prevista não são estatisticamente distintas (Byrne, 2001). 
Portanto o modelo $\mathbf{B}$ apresentou o segundo melhor desempenho em todas as medidas, tanto de ajuste absoluto quanto de ajuste incremental; ele obteve o segundo menor valor de raiz do erro quadrático médio de aproximação RMSEA $=0,03$. Os resultados demonstraram uma covariância não significativa entre as seguintes variáveis latentes: Imitação e Escape $(r=0,19 ; p>0,10)$, entre Moda e Escapar $(r=0,15 ; p>0,10)$, entre Moda e Modelo $(r=0,26 ; p>0,05)$, entre Moda e Imitação $(r=0,15 ; p>0,10)$ e entre Moda e Parafernália $(r=0,00 ; p=\mathrm{NS})$. A Figura 2 apresenta o modelo de segunda ordem, objetivando apresentar as cargas fatoriais padronizadas das dimensões para o construto conexidade.

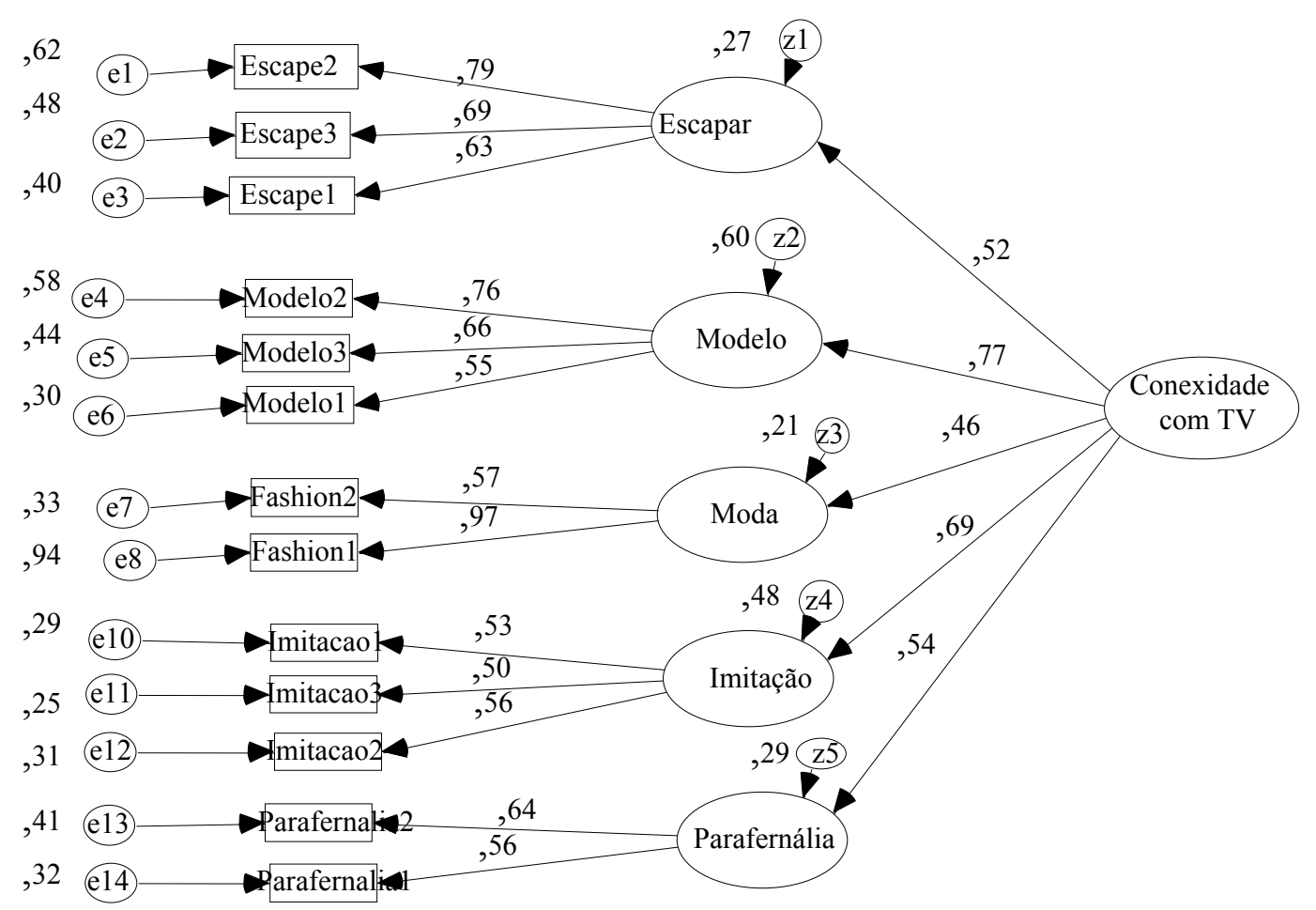

Figura 2. Modelo Estrutural de Segunda Ordem da Conexidade com Programas de TV Nota. Cargas fatoriais dos indicadores $(\lambda)$, erros dos construtos e covariâncias todos padronizados

De acordo com a Tabela 4, o modelo $\mathbf{B}$ seria o mais indicado para representar o construto, uma vez que possui as cinco dimensões propostas originalmente; por isso ele é mostrado. Contudo o modelo $\mathbf{D}$ apresentou ajustamentos ótimos e refuta a aceitação da estrutura B . Diante desses achados, infere-se que a existência empírica do fenômeno teórico conexidade com programas de TV realmente existe, mas a dimensionalidade é questionável. Essa dúvida na estrutura não está apenas nos fatores que refletem o modelo, mas também no número de indicadores que representam as dimensões. Em outras palavras, Costello e Osborne (2005) e Hair et al., (1998) salientam a existência de no mínimo três indicadores por construto. $\mathrm{Na}$ escala original, o número de itens é muito baixo.

Tabela 4

Ajustamentos Confirmatórios dos Modelos Rivais Verificados

\begin{tabular}{clcccccccc}
\hline Modelo & Descrição & $\chi^{2} /$ g.l. & $p$-valor & AGFI & GFI & TLI & CFI & RMSEA & ECVI \\
\hline A & $2^{\text {a }}$. Ordem & 1,17 & $p=0,17$ & 0,88 & 0,92 & 0,95 & 0,96 & 0,04 & 1,05 \\
B & 5 fatores corr. (1 $1^{\text {a }}$ ordem) & 1,09 & $p=0,30$ & 0,89 & 0,93 & 0,98 & 0,98 & 0,03 & 1,04 \\
C & Único fator & 2,85 & $p<0,000$ & 0,74 & 0,81 & 0,50 & 0,59 & 0,12 & 1,88 \\
D & 3 dimensões & 1,01 & $p=0,46$ & 0,87 & 0,94 & 1,00 & 1,00 & 0,01 & 0,88 \\
\hline
\end{tabular}

Nota. Adjusted Goodness-of-Fit [AGFI], Goodness-of-Fit [GFI], Bentler-Comparative-Fit [CFI], Root Mean Square Error of Approximation [RMSEA], Tucker-Lewis Index [TLI], Expected Cross-Validation Index [ECVI]; $\chi^{2} / \mathrm{g} .1 .=$ qui-quadrado por graus de liberdade; $p=$ nível de significância. 
Teste das Proposições. A próxima etapa apresenta os resultados das proposições de pesquisa para a verificação da rede nomológica. A análise de correlação foi utilizada inicialmente para o teste de validade discriminante entre construtos e entre itens, os quais foram apresentados na revisão de literatura. Segundo Russell et al. (2004), a ideia é que uma pequena correlação ou correlação nãosignificativa proporcionaria evidências de validade discriminante e apoiaria as três proposições iniciais de pesquisa.

Nesse sentido, análises de correlações bivariadas foram feitas entre os construtos (média da escala) e entre os itens. Portanto os resultados encontrados foram: conexidade com programas de TV e envolvimento tiveram uma associação de $r=0,19(p<0,05)$, confirmando a $\mathrm{P}_{2 \mathrm{a}}$; numa variação dentro dos itens de $r=0,35(p<0,01)$ até $r=0,00(p=\mathrm{NS})$. Conexidade com programas de TV e atitude tiveram uma correlação geral de $r=0,31(p<0,001)$, numa variação de $r=0,43(p<0,01)$ até $r=0,00$ $(p=\mathrm{NS})$ nos indicadores. Este achado apoia a $\mathrm{P}_{1 \mathrm{a}}$. Por fim, conexidade com programas de TV e Assistir TV no geral não tiveram associação, rejeitando a $\mathrm{P}_{3 \mathrm{a}}$, mas apoiando a $\mathrm{P}_{3 \mathrm{~b}}$. Esses resultados, portanto, suportam as proposições um, dois e três de distinção entre cada conceito.

Adicionalmente, a validade discriminante de duas proposições $\left(\mathrm{P}_{1 b}\right.$ e $\left.\mathrm{P}_{2 \mathrm{~b}}\right)$ foi verificada por meio da sugestão de Fornell e Larcker (1981). Russell et al. (2004) usaram apenas as correlações bivariadas e não essa outra sugestão como complemento. Em outras palavras, o teste consiste na comparação entre a variância média extraída [AVE] de cada construto e o quadrado do coeficiente de correlação entre dois construtos $\left(r^{2}\right)$. De acordo com a Tabela 5, a AVE de conexidade com programas de TV foi maior do que o $r^{2}$ de Envolvimento e de Atitude, confirmando ainda mais as duas primeiras hipóteses

Tabela 5

Matriz de correlação e correlações ao quadrado das variáveis

\begin{tabular}{lcccccccccc}
\hline Variável & $\boldsymbol{M}$ & Envolvimento & Marca & Atitude & Escapar & Modelo & Moda & \multicolumn{1}{l}{ Imitação } & Parafernália Conexidade \\
\hline Envolvimento & 3,15 & $\mathbf{( 0 , 5 0 )}$ & 0,06 & 0,02 & 0,05 & 0,00 & 0,00 & 0,00 & 0,03 & 0,04 \\
Marca & 3,41 & $0,254^{* *}$ & $\mathbf{( 0 , 5 1 )}$ & 0,05 & 0,25 & 0,25 & 0,14 & 0,07 & 0,21 & 0,45 \\
Atitude & 3,74 & 0,146 & $0,230^{* *}$ & $\mathbf{( 0 , 5 8 )}$ & 0,15 & 0,03 & 0,04 & 0,00 & 0,01 & 0,10 \\
Escapar & 3,65 & $0,229^{*}$ & $0,504^{* * *}$ & $0,387^{* * *}$ & $\mathbf{( 0 , 5 0 )}$ & 0,11 & 0,03 & 0,00 & 0,06 & 0,40 \\
Modelo & 3,11 & 0,087 & $0,501^{* * *}$ & $0,184^{*}$ & $0,327 * * *$ & $\mathbf{( 0 , 4 4 )}$ & 0,07 & 0,10 & 0,06 & 0,53 \\
Moda & 3,48 & 0,109 & $0,376^{* * *}$ & $0,199^{*}$ & $0,184^{*}$ & $0,265^{* *}$ & $\mathbf{( 0 , 5 2 )}$ & 0,06 & 0,00 & 0,28 \\
Imitação & 2,21 & 0,022 & $0,264^{* *}$ & 0,082 & 0,170 & $0,324 * * *$ & $0,239^{* *}$ & $\mathbf{( 0 , 4 4 )}$ & 0,07 & 0,42 \\
Parafernália & 2,62 & $0,185^{*}$ & $0,459^{* * *}$ & 0,121 & $0,248^{* *}$ & $0,249 * *$ & 0,091 & $0,26 * *$ & $\mathbf{( 0 , 3 6 )}$ & 0,33 \\
Conexidade & 3,01 & $0,199^{*}$ & $0,674 * *$ & $0,313 * *$ & $0,633^{* *}$ & $0,728^{* *}$ & $0,528^{* *}$ & $0,64 * *$ & $0,577 * *$ & $\mathbf{( 0 , 3 7 )}$ \\
\hline
\end{tabular}

Nota. Correlações ${ }^{*} p<0,05 ; *^{*} p<0,01 ; * * * p<0,001$; entre parênteses indica o AVE; $M=$ média; diagonal superior são as correlações ao quadrado; Cálculo de correlação Pearson na diagonal inferior feito via média dos itens.

Por consequência, passou-se para o exame da quarta proposição. Esperava-se que pessoas com mais conexidade com programas de TV tivessem uma (P4a) maior interação social; (P4b) um maior censo de comunidade de marca; e (P4c) uma maior rede de relacionamentos de amigos (variável contínua). Para o teste, a variável conexidade com programas de TV foi recodificada em baixa, média e alta conexidade (33\%). Os resultados da análise de variância com Tukey indicaram que pessoas mais conexas com programas de TV diferem das pessoas menos conexas no que tange à interação social. Todavia pessoas de baixa conexidade com programas de TV têm interação social maior do que pessoas altamente obcecadas $\left(M_{\text {baixa }}=3,80\right.$ vs $\left.M_{\text {alta }}=2,66 ; F=5,903 ; p<0,01\right)$, indicando o contrário da $\mathrm{P}_{4 \mathrm{a}}$. Uma análise de correlação também indicou que quanto mais conexidade menos interação social $(r=-0,31 ; p<0,001)$, assim a proposição $\mathrm{P}_{4 \mathrm{a}}$ não foi confirmada. 
Em segundo plano, a análise de variância indicou que $\left(\mathrm{P}_{4 b}\right)$ pessoas com mais conexidade com programas de TV diferem significativamente das pessoas com menos conexidade para com maior censo de comunidade de marca $\left(M_{\text {baixa }}=2,40 ; M_{\text {média }}=3,39 ; M_{\text {alta }}=4,45 ; F=37,361 ; p<0,001\right)$. A ponderação ocorre no ponto em que o alto grau de conexidade indica maior consumo, maior adoração, mais indicação e maior amor do produto show de TV. Este achado corrobora a proposição $\mathrm{P}_{4 \mathrm{~b}}$.

Por fim, o teste Kurskal Wallis indicou que $\left(\mathrm{P}_{4 \mathrm{c}}\right)$ pessoas com mais conexidade com programas de TV não diferem significativamente das pessoas menos conexas para uma maior rede de relacionamentos $\left(M_{\text {baixa }}=59\right.$ vs. $\left.M_{\text {média }}=63 v s . M_{\text {alta }}=54 ; p=\mathrm{NS}\right)$, ou seja, resultado contrário ao esperado. Todavia esse resultado está na mesma linha do achado sugerido pela ANOVA na $\mathrm{P}_{4 \mathrm{a}}$. Portanto nota-se que maior conexidade com programas de televisão parece criar menor associação social e menor rede de amigos; assim, por consequência, menor inclusão social.

Poder de Explicação do Construto. Completando o trabalho de validade, foi feita uma análise de regressão do tipo hierárquico, verificando o poder de predição do construto conexidade . Em cada um dos três modelos ponderados, atitude, envolvimento e visualização geral da televisão foram entradas como variáveis independentes e conexidade com programas de TV foi adicionada em segundo momento também como variável independente. O objetivo foi testar a habilidade preditiva da conexidade, observando o melhoramento dos ajustes do modelo entre a comparação do primeiro passo versus o segundo passo (mudança de $\Delta \mathrm{R}^{2}$ ). As variáveis dependentes de cada uma das três análises de regressão foram: comunidade de marca, interação social e tamanho da rede de amigos.

De acordo com a Tabela 6, os resultados indicaram que no modelo $\mathbf{A}$ atitude foi preditora de interação social e que quando inserida a conexidade com programas de $\mathrm{TV}$ o $\mathrm{R}^{2}$ do modelo aumentou significativamente, demonstrando o poder desta última em explicar negativamente o senso de interação social. No modelo $\mathbf{B}$, os achados ainda sugeriram que atitude e envolvimento foram preditoras de comunidade de marca e quando inserida a conexidade o $\mathrm{R}^{2}$ do modelo aumentou significativamente, demonstrando o poder desta última em explicar o senso de comunidade da marca do programa de TV da qual o telespectador gosta. Posteriormente, na última análise, $\mathbf{C}$, não houve relação significativa entre as variáveis exógenas e aumento de rede de amigos (network). Em resumo, os achados da análise de regressão hierárquica corroboram ainda mais os testes de diferenças de médias ANOVA e Kurskal Wallis, sugerindo o suporte exclusivamente para a proposição $\left(\mathrm{P}_{2 \mathrm{~b}}\right)$.

Tabela 6

\section{Análise de regressão hierárquica}

\begin{tabular}{lcccccc}
\hline \multicolumn{1}{c}{ Dependente } & Conexidade & Atitude & Envolvimento & TV Geral & $\mathbf{R}^{\mathbf{2}}$ & $\boldsymbol{\Delta R}^{\mathbf{2}}$ Diferença \\
\hline (a) Interação Social & $\beta=-0,27^{* *}$ & $\beta=-0,20^{*}$ & $\beta=-0,02$ & $\beta=-0,11$ & 0,03 & $0,06^{* *}$ \\
(b) Comunidade Marca & $\beta=0,64^{* * *}$ & $\beta=0,20^{*}$ & $\beta=0,21^{*}$ & $\beta=0,08$ & 0,09 & $0,36^{* * *}$ \\
(c) Network-rede & $\beta=0,03$ & $\beta=-0,11$ & $\beta=-0,07$ & $\beta=-0,06$ & NS & NS \\
\hline
\end{tabular}

Nota. ${ }^{*} p<0,05 ;{ }^{*} p<0,01 ; * * * p<0,001$; A diferença de $\Delta \mathrm{R}^{2}$ (change) corresponde a diferença significativa de aumento no $\mathrm{R}^{2}$ com a inserção do construto conexidade; NS = não significativo; betas padronizados; VIF não indicou colinearidade; $\mathrm{R}^{2}=$ ajustado

Por fim, vale salientar que o resultado contrário de conexidade com programas de TV e interação social também foi notado no modelo de regressão, uma vez que o beta foi negativo. Os resultados indicam que as pessoas não consomem muitos produtos em termos de comunidade de marca (talvez por não existirem ou existirem poucos, como, por exemplo, os produtos do programa Pânico) e que pessoas mais conexas com programas de TV têm uma rede de relacionamentos menor do que seus compatriotas.

Validação Cruzada. Alguns autores sugerem a validação cruzada para aferir a confiabilidade de uma escala (Floyd \& Widaman, 1995). O objetivo é verificar se a estrutura varia dentro de algumas alterações na amostra. Por exemplo, obter aleatoriamente $50 \%$ da amostra principal e fazer a análise 
fatorial exploratória novamente, pressupondo que o resultado será idêntico ao feito com a amostra total. Esta técnica permite examinar se a estrutura identificada se repete, quando investigada em uma segunda amostra criada artificialmente, conforme salientam Laros e Puente-Palacios (2004). A sua utilização é importante, tanto para soluções fatoriais exploratórias como para soluções fatoriais confirmatórias, nesse último caso empregando-se análises de invariância em multigrupos (Arbuckle, 1997; Kline, 1998). Neste artigo, devido ao tamanho limitado da amostra, não foi possível verificar a validade cruzada do instrumento. Assim, sugere-se esse processo em futuro estudo.

\section{CONSIDERAÇÕES FINAIS}

O artigo buscou verificar a escala de conexidade com programas de TV de Russell et al. (2004). Inicialmente, o conceito teórico de conexidade por televisão existe e é multidimensional. Dentro das cinco dimensões hipotetizadas, Fuga - a qual objetiva mostrar ao consumidor que ele pode fugir dos problemas reais da vida assistindo a TV - mostrou-se a de maior autovalor, explicando sozinha $25 \%$ da estrutura como um todo.

Segundo, excluiu-se a dimensão Aspiração do instrumento. Este resultado evidencia que a amostra não percebeu necessidade de aspiração de um dia estar no show ou encontrar seu protagonista. Por exemplo, eu poderia ser um dia um ator(atriz) no show da Xuxa.

Terceiro, possuir alto envolvimento é diferente de uma conexidade por assistir de TV e essa distinção se aplica também à atitude e horas assistindo TV. Os resultados adicionais feitos por meio da sugestão de Fornell e Larcker (1981) também mostraram essa discriminação entre as variáveis. Em outras palavras, ficar horas e horas assistindo a TV não significa que o indivíduo gerou uma conexidade com o programa.

Uma quarta conclusão ocorre, pois se esperava que pessoas com maior conexidade por programas de TV tivessem maior interação social com outros colegas que assistem ao mesmo show. Isso não ocorreu e o inverso foi demonstrado, indicando que possuir uma alta conexidade gera menor interação social e não mais amigos por meio dos shows televisivos. Adicionalmente, os resultados indicaram que pessoas de maior conexidade diferem significativamente das pessoas de menor grau para com um maior censo de comunidade de marca. A comunidade de marca objetivava recomendar, amar, orgulhar, sugerir etc., o programa de TV em específico. Neste sentido, pessoas com maior conexidade tinham médias diferentes no quesito comunidade de marca do que pessoas com menor grau.

Por fim, uma conclusão não esperada apontou a possível explicação: possuir maior conexidade com TV gera menor interação social. Em outras palavras, isso quer dizer que pessoas mais obcecadas em TV não diferem significativamente das pessoas menos obcecada no quesito maior rede de relacionamentos (network). Esse achado também é encontrado na tabela da análise de regressão.

Em resumo, os resultados do trabalho corroboram a existência do construto conexidade de Russell e Puto (1999). Deste modo, o artigo auxilia na contribuição do desenvolvimento de teorias no campo do comportamento do consumidor, cuja tese de consumo obsessivo por TV foi examinada. Não obstante, esse mesmo auxílio apóia ainda mais o conceito generalização empírica de fenômenos para a evolução da teoria em marketing e comportamento do consumidor (Bass \& Wind, 1995; Hunt, 1991), dada a validade da conexidade por programas de TV em outra cultura.

\section{Pesquisas Futuras}

Um artigo futuro pode criar novos indicadores para as dimensões Imitação e Parafernália, uma vez que apresentaram baixa confiabilidade. Neste novo trabalho autores podem utilizar-se de pesquisas qualitativas e entrevistas para validar a semântica da escala. Segundo, trabalhos futuros poderiam analisar o grau conexidade em outros objetos. Por exemplo, por que pessoas têm conexão (uso 
demasiado) com celular, com Internet ou com carros? Terceiro, pesquisas futuras poderiam verificar o instrumento de Russell et al. (2004) em outras culturas (Européia, Asiática). Por exemplo, será que outros países de primeiro mundo, cuja programação televisiva é menos censurada, existe a mesma estrutura da escala encontrada no Brasil ou nos EUA? Pesquisas podem desenvolver mais a rede nomológica, aqui sugerida, relacionando a escala de conexidade com a de experiências depressivas. Rude e Burnham (1995) fizeram este exame, mas trabalhando com outro instrumento de conexidade. Quinto, a conexidade com programas de TV pode gerar maior autonomia nos consumidores para desenvolverem uma rede de relacionamento pessoal. Em outras palavras, a conexidade com uma novela pode gerar satisfação no indivíduo, uma vez que ele se engaja com maior dedicação em uma relação interpessoal positiva com outros que assistem ao mesmo programa. Nesse sentido, uma interação entre conexidade e a autonomia (assertividade e consciência) pode existir, como foi encontrado por Bekker, Croon, van-Balkom e Vermee (2008). Por fim, a conexidade com programas de TV pode ter relação com a conexidade-(des)conexidade gerada pela atitude de um apelo da propaganda. Em outras palavras, Wang e Mowen (1998) encontraram maior efeito da comunicação em marketing, quando existe congruência entre a conexidade com o programa de TV vs conexidade(des)conexidade de um tema de propaganda.

Artigo recebido em 04.11.2008. Aprovado em 10.08.2009.

\section{NOTAS}

\footnotetext{
${ }^{1}$ Original em inglês: Connectedness Scale.

${ }^{2}$ Original em inglês: Escape, Modeling, Fashion, Imitation, Aspiration e Paraphernalia.

${ }^{3}$ Os valores alphas, confiabilidade composta e média da variância extraída para as escalas foram computados. Assim, a comunidade de marca $(\alpha=0,79)$, atitude $(\alpha=0,85)$ e envolvimento $(\alpha=0,82)$ obtiveram bons valores. Adicionalmente, no que tange aos construtos atitude $(\mathrm{CR}=0,85 ; \mathrm{AVE}=0,58)$, envolvimento $(\mathrm{CR}=0,82 ; \mathrm{AVE}=0,50)$ e comunidade de marca $(\mathrm{CR}=0,79 ; \mathrm{AVE}=0,51)$, todos os valores mínimos de confiabilidade foram atingidos.

${ }^{4}$ Os indicadores estão ordenados. Por exemplo, as três fases de Espace correspondem aos itens 1,2 e 3, respectivamente a escala original.
}

\section{REFERÊNCIAS BIBLIOGRÁFICAS}

Aaker, D., Day, G., \& Kumar, V. (2005). Pesquisa de marketing. Atlas: São Paulo.

Arbuckle, J. L. (1997). AMOS user's guide: Version 3.6. Chicago: SPSS.

Bagozzi, R. P., \& Philips, L.W. (1982). Representing and testing organizational theories: a holistic construal. Administrative Science Quarterly, 27(3), 459-489.

Barwise, P. T., \& Ehrenberg, A. S. C. (1987). The liking and viewing of regular TV series. Journal of Consumer Research, 14(3), 63-70.

Bass, F. M., \& Wind, J. (1995). Introduction to the special issue: empirical generalizations in marketing. Marketing Science, 14(3), 1-5.

Bekker, M. M., Croon, M. A., van-Balkom, E. G. A., \& Vermee, J. B. G. (2008). Predicting individual differences in autonomy-connectedness: the role of body awareness, alexithymia, and assertiveness. Journal of Clinical Psychology, 64(6), 747-765. 
Belk, R. W. (1988). Possessions and the extended self. Journal of Consumer Research, 15(2), 139-168.

Belk, R. W. (1992). Moving Possessions: an analysis based on personal documents from the 18471869 Mormon Migration. Journal of Consumer Research, 19(3), 339-54.

Byrne, B. (2001). Structural equation modeling with AMOS: basic concepts, applications and programming (Multivariate Book Series, Lawrence Erbaum Associate). New Jersey: Lawrence Earbaum Associates.

Cacioppo, J. T., Gardner, W. L., \& Berntson, G. G. (1997). Beyond bipolar conceptualizations and measures: the case of attitudes and evaluative space. Personality and Social Psychology Review, $1(1), 03-25$.

Churchill, G. A., Jr. (1996). Marketing research: methodological foundations (3rd ed.). Chicago: The Dryden Press.

Costello, A. B., \& Osborne, J. W. (2005). Best practices in exploratory factor analysis: four recommendations for getting the most from your analysis. Practical Assessment, Research \& Evaluation, 10(7), 1-9.

Dholakia, U. M. (1997). An Investigation of the Relationship between Perceived Risk and product involvement. Advances in Consumer Research, 24(1), 159-167.

Engel, J. F., Blackwell, R. D., \& Miniard, P. (2000). Comportamento do consumidor. Rio de Janeiro: LTC.

Enzmann, D. (1997). RanEigen: a program to determine the parallel analysis criterion for the number of principal components. Applied Psychological Measurement, 21(3), 232.

Floyd, F. J., \& Widaman, K. F. (1995). Factor analysis in the development and refinement of clinical assessment instruments. Psychological Assessment, 7(3), 286-299.

Fornell, C., \& Larcker, D. F. (1981). Evaluating structural equations models with unobservable variables and measurement error. Journal of Marketing, 18(1), 39-50.

Fournier, S. (1998). Consumers and their brands: developing relationship theory in consumer research. Journal of Consumer Research, 24(3), 343-373.

Hair, J. F., Anderson, R. E., Tatham, R. L., \& Black, W. (1998). Multivariate data analysis (5th ed.). New Jersey: Prentice Hall.

Hunt, S. (1991). Modern marketing theory: critical issues in the philosophy of marketing science. Ohio: South-Western Publishing Co.

Kagami, M. (2006). Futuro da sociedade TI: convergência ou coexistência? Recuperado em 07 abril, 2006, de http://www.cidade.usp.br/arquivo/artigos/index1101.php

Kline, R. B. (1998). Principles and practice of structural equation modeling. New York: Guildford Press.

Kozinets, R. V. (2001). Utopian enterprise: articulating the meanings of star trek's culture of consumption. Journal of Consumer Research, 28(2), 67-68.

Krugman, H. E. (1977). Memory without recall, exposure without perception. Journal of Advertising Research, 17(4), 7-12.

Laros, J., \& Puente-Palacios, K. E. (2004). Validação cruzada de uma escala de clima organizacional. Estudos de Psicologia, 9(1), 113-119. 
Laurent, G., \& Kapferer, J. N. (1985). Measuring consumer involvement profiles. Journal of Marketing Research, 22(1), 41-53.

Levy, S. (1962). Phases in Changing Interpersonal relations. Merrill-Palmer Quarterly of Behavior ad Development, 8(2), 121-128.

Maccoby, E. E., \& Wilson, W. C. (1957). Identification and observational learning from films. Journal of Abnormal and Social Psychology, 55(1), 76-87.

Macinnis, D. J., \& Whan, P. C. (1991). The differential role of characteristics of music on high and low involvement consumers' processing of ads. Journal of consumer Research, 18(2), 161-174.

Malhotra, N. (2000). Pesquisa de marketing. Porto Alegre: Bookman.

McAlexander, J. H., Schouten, J. W., \& Koening, H. (2002). Building brand community. Journal of Marketing, 66(1), 38-54.

Murry, J. P., Lastovicka, J. L., \& Singh, S. N. (1992). Feeling and liking responses to television programs: an examination of two explanations for media-context effects. Journal of Consumer Research, 18(1), 441-451.

Oliveira, E., Sanvitto, G., Gus, P., \& Zelmanowicz, R. U. (2009). Seu filho está assistindo televisão demais? Recuperado em 28 junho, 2010, de http://www.abcdasaude.com.br/artigo.php?703

Park, W. C., \& McLung, G. W. (1986). The effect of TV program involvement on involvement with commercials. Advances in Consumer Research, 13(1), 544-548.

Peter, P. J. (1981). Construct Validity: a review of basic issues and marketing practices. Journal of Marketing Research, 18(1), 133-145.

Richins, M. L., \& Bloch, P. H. (1986). Research in brief after the new wears off: the temporal context of product involvement. Journal of Consumer Research, 13(2), 280-286.

Rude, S. S., \& Burnham, B. L. (1995). Connectedness and neediness: factors of the DEQ and SAS dependency scales. Cognitive Therapy and Research, 19(3), 323-340.

Russell, C. A., Norman, A. T., \& Heckler, S. E. (2004). The consumption of Television programming: development and validation of the connectedness scale. Journal of Consumer Research, 31(1), 150-161.

Russell, C. A., \& Puto, C. P. (1999). Rethinking television audience measures: an exploration into the construct of Audience connectedness. Marketing Letters, 10(4), 393-407.

Vieira, V. A. (2009). Is the need to evaluate a real consumer need? Brazilian Administration Review, 6(2), 153-172. Recuperado em 26 junho, 2010, de http://www.anpad.org.br/periodicos/arq_pdf/a_851.pdf

Vieira, V. A., \& Slongo, L. A. (2007). An inventory of the characteristics of the marketing scales created and tested in Brazil. Revista de Administração Mackenzie, 8(4), 11-34.

Wang, C. L., \& Mowen, J. C. (1998). The separateness-connectedness self-schema: scale development and application to message construction. Psychology and Marketing, 14(2), 185-207.

West, S., Finch, J., \& Curran, P. (1995). Structural equation models with nonnormal variables: problems and remedies. In R. Hoyle (Ed.), Structural equation modeling: concepts, issues and applications (pp. 65-81). London: SAGE.

William, S. R. (1993). The effects of mood, involvement and quality of store experience on shopping intentions. Journal of Consumer Research, 20(2), 271-281. 
Zaichkowsky, J. L. (1985). Measuring the involvement construct. Journal of Consumer Research, 12(3), 341-352.

Zwick, R., \& Velicer, W. F. (1986). Comparison of five rules for determining the number of components to retain. Psychological Bulletin, 99(3), 432-442. 


\section{ANEXO I}

\section{Escala de Conexidade por programas de TV(4)}

Escapar: Assistir ao programa __é um alívio para mim; O programa me ajuda a esquecer dos problemas do dia-a-dia; Se eu estou de mau humor, assistir ao programa me faz sentir melhor Moda: Eu gosto das roupas que eles (personagens) usam em __ Eu gosto do estilo do corte de cabelo das personagens em__ Eu frequentemente compro os estilos de roupas que vejo em Imitação: Eu imito os gestos e expressões faciais das personagens de __ ; Quando estou falando com as pessoas, às vezes me pego dizendo frases de __; Eu tento falar como as personagens de Modelar: Eu aprendo a lidar com situações reais da vida assistindo _ _; Eu obtenho idéias de sobre como interagir na minha própria vida; Eu relaciono o que acontece em _ com minha própria vida; Aspiração: Eu poderia gostar de ser um(a) ator(atriz) em __ ; Eu poderia gostar de encontrar as personagens de ___ Parafernália: Eu tenho objetos que se relacionam com __; Eu leio livros, caso eles sejam relacionados com

\section{ANEXO II}

\section{Demais escalas utilizadas}

Atitude: Gosto/não gosto; bom/ruim; agradável/desagradável. Envolvimento: importante/nãoimportante; Interessante/chato; Querido/não-querido; provoca interesse/não provoca interesse; faz diferença/não faz diferença; Comunidade de Marca: Eu recomendaria para os outros; Eu amo o programa ___ É muito divertido assistir __ Eu tenho orgulho desse programa; Se existisse a possibilidade de assistir a outro programa, eu assistiria um igual ao meu; O programa _ é de alta qualidade; Assistir _ acaba criando um sentimento de parentesco c/ outras pessoas. 